\title{
A Method to Remotely Track a Magnetic Target Using a Scalar Magnetometer Array
}

\author{
Xiaojun Zhang, ${ }^{1}$ Liming Fan, ${ }^{1,2}$ Peng Cheng, ${ }^{1}$ Chunlei Chen, ${ }^{1}$ Xuejun Liu, ${ }^{1}$ \\ and Chong Kang ${ }^{1,2}$ \\ ${ }^{1}$ College of Science, Harbin Engineering University, Harbin 150001, China \\ ${ }^{2}$ College of Mechanical and Electrical Engineering, Harbin Engineering University, Harbin 150001, China \\ Correspondence should be addressed to Chong Kang; kangchongheu@163.com
}

Received 15 June 2017; Revised 18 October 2017; Accepted 1 November 2017; Published 19 December 2017

Academic Editor: Paolo Bruschi

Copyright (c) 2017 Xiaojun Zhang et al. This is an open access article distributed under the Creative Commons Attribution License, which permits unrestricted use, distribution, and reproduction in any medium, provided the original work is properly cited.

\begin{abstract}
The orientation of a vector magnetic sensor can affect the measurement accuracy of magnetic anomaly, thereby increasing the localization error of a magnetic target. Compared with vector magnetic sensor, the measurement of the scalar magnetic sensor is almost not influenced by its orientation. Therefore, we present a method for tracking the magnetic target with a static scalar magnetometer array. In this study, the magnitude of the target's magnetic moment is a key parameter. We isolate it and formulate an optimization problem based on it to estimate the position and magnetic parameters of the target. To calculate the solution of this optimization problem, a dedicated particle swarm optimization (PSO) algorithm is developed. Then, we define a quality index to evaluate the solution calculated by the optimization problem. The proposed method was validated by the simulation and the real data collected when an SUV car was passing by the array on a straight path. The results show that the tracked trajectory is very close to the true trajectory and the quality index can be used as a criterion to allow accepting or rejecting the localization of the target.
\end{abstract}

\section{Introduction}

A target containing ferromagnetic material can be detected by magnetic sensors due to a magnetic field generated by it. Along with the development of the techniques for the magnetic anomaly detection, researchers have proposed several methods for target localization or tracking [1-9]. Usually, we can describe the target with six parameters $\left\{x, y, z, m_{x}\right.$, $\left.m_{y}, m_{z}\right\}$. A vector sensor can provide much valuable target information. In order to calculate six parameters of the target, at least two vector magnetometers are needed to build six nonlinear functions. Thus, a sensor array is widely used to locate the magnetic target. Wynn [1] proposed a method of magnetic dipole localization based on the magnetic gradient. Nara et al. [2] obtained a localization formula for magnetic dipole localization by magnetic vectors and their spatial gradients. In this method, the accuracy of localization is highly sensitive to noise in magnetic anomaly field vector data. The measurement data consist of the ambient geomagnetic field and the magnetic fields produced by the target. Therefore, it is hard to obtain the accurate magnetic anomaly field from vector magnetometers in practice. Generally, the gradient of the ambient geomagnetic field is small, and the gradient tensor of the magnetic measurement can reflect the magnetic anomaly of the target. In order to overcome the effect of the geomagnetic field, magnetic gradient tensor arrays comprising multiple vector magnetometers were proposed to locate the magnetic target $[4,5,10-12]$. Liu and Wang [10] proposed a method for locating the magnetic target using a 3-axis magnetic sensor array. Gang et al. [5] presented a rotationally invariant method for locating a magnetic target using a magnetic gradient tensor system. Despite the obvious advantages in localization with the gradient tensor, challenges exist with gradient tensor measurements. The reasons are as follows: (1) Rotational vibrations of the array will lead to the orientation error of each vector 


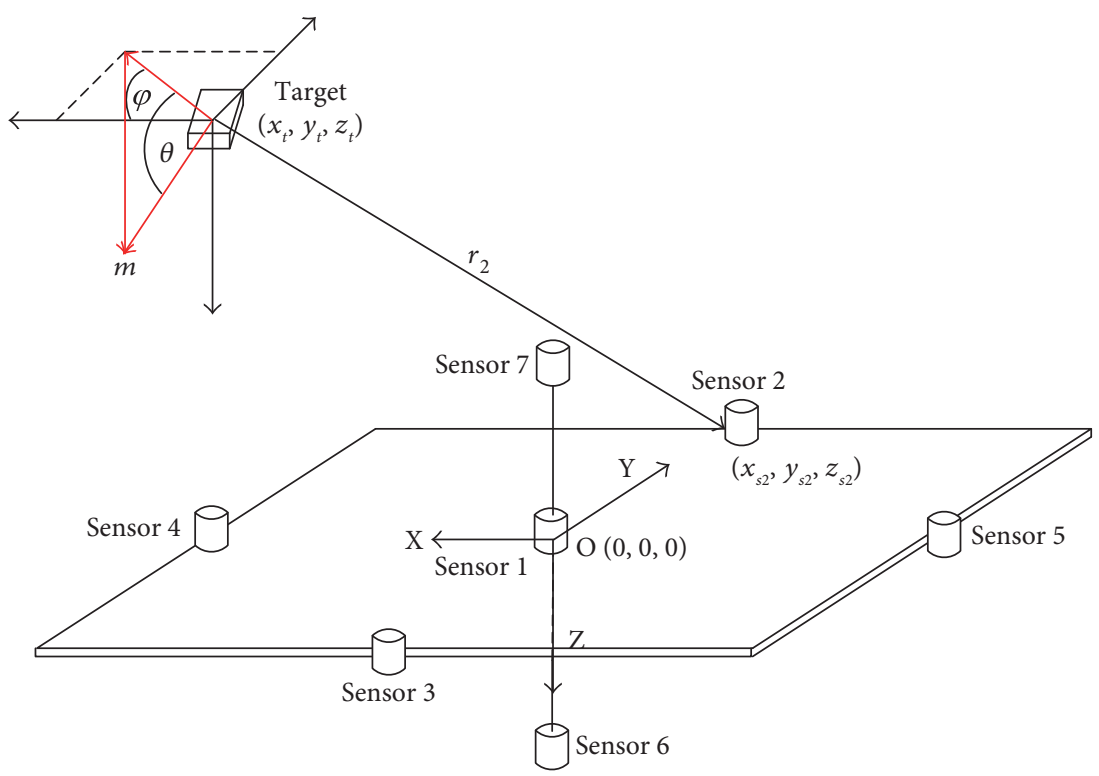

FIGURE 1: Schematic diagram of the scalar magnetometer array.

sensor. (2) The strict alignment for the vector sensors in the array is very difficult. (3) Three axes of the vector sensor are not exactly perpendicular. These reasons will affect the magnetic anomaly measurements [13].

However, a scalar magnetic sensor such as an optically pumped magnetometer has an important advantage in that it is relatively insensitive to its orientation. The optically pumped magnetometer does not work in only two orientations when the optical axis is parallel to the ambient field or perpendicular to the ambient field. Therefore, it can work well for a large range of angles. In many location methods, the Euler deconvolution method [14-16], which is important for magnetic data interpretation, is used to determine the depth and location of the static target using a moving sensor. In this method, magnetic field grid data (profile or map) are used to determine the position or boundary [17]. Thus, magnetic field data within the region need to be measured.

In this paper, we focus on using a static scalar magnetometer array instead of a stationary array of vector magnetometers to track a moving magnetic target. We present a method for determining the parameters of the moving target. In this method, we isolate the magnitude of the magnetic moment and formulate an optimization problem based on it to estimate the position and magnetic parameters of the target.
This can reduce the number of unknown parameters of the optimization problem. Then, we develop a dedicated PSO algorithm to calculate the solution of this optimization problem without the initial parameter values. The PSO algorithm cannot always converge to a global optimal solution in every calculation. Thus, we define a quality index as the criterion for accepting or rejecting the calculated solution in the PSO algorithm.

\section{Localization Theory Based on Scalar Magnetometer Array}

The magnetic target can be considered a magnetic dipole when the distance between it and a magnetic sensor is 3 times larger than the size of the dipole's largest dimension [18]. The magnetic field vector $\mathbf{B}_{\mathrm{A}}$ generated by the dipole is a function of the distance $r$ between the target and the sensor and the magnetic moment $m$ of its own. We build an array with seven scalar magnetometers. The center of the array is located at the origin of a Cartesian coordinate system with the $\mathrm{X}$-axis toward to the geographical north, the Y-axis toward to the geographical east, and $\mathrm{Z}$-axis downward to the ground, as shown in Figure 1. In the coordinate system, the magnetic field generated by the target can be described as follows:

$$
\mathbf{B}_{\mathrm{A}}=\left[\begin{array}{l}
\mathbf{B}_{\mathrm{A} x} \\
\mathbf{B}_{\mathrm{A} y} \\
\mathbf{B}_{\mathrm{A} z}
\end{array}\right]=\frac{\mu_{0} m}{4 \pi\|r\|^{3}}\left(3 \frac{x \cos (\theta) \cos (\varphi)+y \cos (\theta) \sin (\varphi)+z \sin (\theta)}{\|r\|^{2}}\left[\begin{array}{l}
x \\
y \\
z
\end{array}\right]-\left[\begin{array}{c}
\cos (\theta) \cos (\varphi) \\
\cos (\theta) \sin (\varphi) \\
\sin (\theta)
\end{array}\right]\right),
$$

where $\mu_{0}=4 \pi \times 10^{-7} \mathrm{H} / \mathrm{m}$ is the permeability of free space. $\|r\|$ is the distance from the target to the sensor. $m$ is the magnitude of the magnetic moment. $\theta$ is the rotation angle from the magnetic moment vector toward the $\mathrm{X}-\mathrm{Y}$ plane, and $\varphi$ is the rotation angle from the positive $\mathrm{X}$-axis in the $\mathrm{X}-\mathrm{Y}$ plane. $x=x_{t}-x_{s}, y=y_{t}-y_{s}$, and $z=z_{t}-z_{s}$. 
In practice, the measured magnetic field, $\mathbf{B}_{m}$, is the vector sum of the ambient geomagnetic field $\mathbf{B}_{\mathrm{E}}$ and the target magnetic field $\mathbf{B}_{\mathrm{A}}[19]$ :

$$
\mathbf{B}_{m}=\mathbf{B}_{\mathrm{E}}+\mathbf{B}_{\mathrm{A}}
$$

The magnetic field generated by the remote target is far less than the ambient geomagnetic field at the measurement point. According to the approximation, we can rewrite $\mathbf{B}_{m}$ in the scalar form as follows:

$$
\begin{aligned}
\left|\mathbf{B}_{m}\right| & =\sqrt{\left(\mathbf{B}_{\mathrm{E}}+\mathbf{B}_{\mathrm{A}}\right)^{2}} \\
& \cong \sqrt{\left|\mathbf{B}_{\mathrm{E}}\right|^{2}+2 \mathbf{B}_{\mathrm{E}} \cdot \mathbf{B}_{\mathrm{A}}} \\
& \cong\left|\mathbf{B}_{\mathrm{E}}\right|+\frac{\mathbf{B}_{\mathrm{E}}}{\left|\mathbf{B}_{\mathrm{E}}\right|} \cdot \mathbf{B}_{\mathrm{A}} .
\end{aligned}
$$

Hence, the magnetic anomaly $\Delta B$ measured by a scalar magnetometer can be given as follows:

$$
\begin{aligned}
\Delta B & =\left|\mathbf{B}_{m}\right|-\left|\mathbf{B}_{\mathrm{E}}\right| \\
& =\mathbf{u} \cdot \mathbf{B}_{\mathrm{A}} \\
& =\left[\begin{array}{c}
\cos I \cos D \\
\cos I \sin D \\
\sin I
\end{array}\right]^{T} \cdot\left[\begin{array}{c}
\mathbf{B}_{\mathrm{A} x} \\
\mathbf{B}_{\mathrm{A} y} \\
\mathbf{B}_{\mathrm{A} z}
\end{array}\right],
\end{aligned}
$$

where $\mathbf{u}$ denotes the direction vector of $\mathbf{B}_{\mathrm{E}} . I$ and $D$ denote the inclination and declination of the ambient geomagnetic field, respectively. It is seen from (4) that the magnetic anomaly can be regarded as the projection of $\mathbf{B}_{\mathrm{A}}$ on $\mathbf{B}_{\mathrm{E}}$.

When the magnitude of the magnetic anomaly generated by the target is small, geomagnetic field variation will affect the localization. Generally, the broad characteristics of geomagnetic field variation are consistent over the local region. We can consider that its effect is the same for the measurement of each magnetometer in the array, shown in Figure 1. Therefore, we can eliminate the effect of geomagnetic field variation through the subtraction with two magnetometers and obtain an equation as follows:

$$
\begin{aligned}
\Delta B_{i j} & =\mathbf{u}_{i} \cdot \mathbf{B}_{\mathrm{A} i}-\mathbf{u}_{j} \cdot \mathbf{B}_{\mathrm{A} j} \\
& =m\left(\mathbf{G}_{i} \cdot \alpha_{i}\left(\beta_{i} \mathbf{R}_{i}-\mathbf{Q}\right)-\mathbf{G}_{j} \cdot \alpha_{j}\left(\beta_{j} \mathbf{R}_{j}-\mathbf{Q}\right)\right) \\
& =m f_{i j}\left(x_{t}, y_{t}, z_{t}, \theta, \varphi\right) \quad(i, j=1, \ldots, 7 ; i \neq j),
\end{aligned}
$$

where $i$ and $j$ are the indices of individual sensors in the array.

$$
\begin{aligned}
& \alpha=\frac{\mu_{0}}{4 \pi\|r\|^{3}}, \\
& \beta=3 \frac{x \cos (\theta) \cos (\varphi)+y \cos (\theta) \sin (\varphi)+z \sin (\theta)}{\|r\|^{2}}, \\
& r=\left[\begin{array}{l}
x \\
y \\
z
\end{array}\right], \\
& \mathbf{G}=\left[\begin{array}{c}
\cos I \cos D \\
\cos I \sin D \\
\sin I
\end{array}\right] \\
& \mathbf{Q}=\left[\begin{array}{c}
\cos (\theta) \cos (\varphi) \\
\cos (\theta) \sin (\varphi) \\
\sin (\theta)
\end{array}\right] .
\end{aligned}
$$

There are six parameters in (5), which can be combined into a single six-dimensional vector as $\mathbf{p}=\left(x_{t}, y_{t}, z_{t}, \theta, \varphi, m\right)$. Equation (5) is a high-order nonlinear function, which usually is solved by optimization methods. We define an objective error function as

$$
F=\sum_{i, j}\left(\Delta B_{i j}^{m}-\Delta B_{i j}^{c}(\mathbf{p})\right)^{2}
$$

where $\Delta B_{i j}^{m}$ is the magnetic field measured by the $i$ th and $j$ th magnetometers. $\Delta B_{i j}^{c}(\mathbf{p})$ is the magnetic field calculated by (5). It can be seen that (7) represents the mean-squared error between the measured and the calculated magnetic fields. Thus, this is a least square error problem. The parameter vector $\mathbf{p}$ can be solved by minimizing the error through an appropriate optimization algorithm. This is the normal method for tracking the magnetic target.

Because (5) is a high-order nonlinear function, there are some local optimal solutions in the solution space. It is known that the objective function can affect the performance of the algorithm. If the function is simpler and has fewer parameters, the accuracy of the solution calculated by the optimization algorithm is higher. Thus, we isolate the magnitude of the magnetic moment and rewrite (5) as

$$
m=\frac{\Delta B_{i j}}{f_{i j}\left(x_{t}, y_{t}, z_{t}, \theta, \varphi\right)} .
$$

According to (8), we define a new objective error function as

$$
F=\sum\left(\frac{1}{\Delta B_{i j}} f_{i j}\left(x_{t}, y_{t}, z_{t}, \theta, \varphi\right)-\frac{1}{\Delta B_{k l}} f_{k l}\left(x_{t}, y_{t}, z_{t}, \theta, \varphi\right)\right)^{2}
$$

Thus, the new error function has one less parameter than (7). This is good for improving the accuracy of the solution. 
Sometimes, the optimization algorithm cannot jump out from the local optima when trapped in it and the algorithm may fail to give the right global solution. Thus, we should define a criterion to evaluate the solution and determine whether to accept or reject the solution according to the criterion.

Once the parameters $\left(x_{t}, y_{t}, z_{t}, \theta, \varphi\right)$ are calculated by the optimization algorithm, the magnitude of the magnetic moment is easily calculated according to (8). Therefore, we can calculate a series of $m$ according to the different magnetic anomalies $\Delta B_{i j}$, which are measured in the array. Theoretically, if the parameters $\left(x_{t}, y_{t}, z_{t}, \theta, \varphi\right)$ of the target are the true value, the calculated moments $m$ are equal to the same value. However, the measurement error leads to the error of parameters $\left(x_{t}, y_{t}, z_{t}, \theta, \varphi\right)$ calculated by the method. Thus, it is highly unlikely for the calculated moments, $m$, to have the same value. When the parameter errors are small, the calculated moments $m$ are close to the same value. Conversely, when the error of parameters $\left(x_{t}, y_{t}, z_{t}, \theta, \varphi\right)$ is large, the fluctuation of calculated moments $m$ is also large. Therefore, according to the magnetic moment, we define the criterion for the quality of the solution called the quality index as

$$
\mathrm{QI}=\exp \left(-\sum_{p, q}\left(\frac{m_{p}}{m_{q}}-1\right)^{2} \times 10\right) \quad(p \neq q)
$$

The magnitude of the magnetic moment $m$ with different subscripts ( $p$ or $q$ ) denotes the calculated magnitude of the magnetic moment using the different $\Delta B_{i j}$.

It is noted that the expression $\sum_{p, q}\left(\left(m_{p} / m_{q}\right)-1\right)^{2}$ is the squared relative error between the calculated magnitudes of the magnetic moment using the different $\Delta B_{i j}$. The value of this expression is in the range $[0, \infty)$. It is more convenient for the quality index to be in the interval $[0,1]$. Thus, we introduce the exponential function and give the expression of the quality index as (10).

From (10), the closer the values of $m$ calculated by different $\Delta B$ are, the closer the expression $\mathrm{QI}$ is to 1 . This means that the parameters $\left(x_{t}, y_{t}, z_{t}, \theta, \varphi\right)$ of the target calculated by the PSO algorithm are close to the true values. On the contrary, if the parameters $\left(x_{t}, y_{t}, z_{t}, \theta, \varphi\right)$ of the target calculated by the PSO algorithm are the local optimal solution, the fluctuation of calculated moments $m$ is also large. This can cause the value of QI to be far from 1 . Therefore, the quality index can be used as an indicator for determining whether the calculated solution is a global optimal solution.

\section{Localization Algorithm Based on the PSO Algorithm}

There are many conventional algorithms (such as LevenbergMarquardt, Newton-Gauss algorithm, and Fletcher-Powell), which can be used to solve the optimization problem. The Levenberg-Marquardt (L-M) algorithm can be regarded as a combination of the steepest descent method and the Newton-Gauss method. The main advantage of the L-M algorithm is its rapidity and its well-known and reliable implementation [20]. However, the L-M algorithm cannot guarantee convergence to a global minimum unless the initial parameter guesses are appropriate [21]. In the tracking system, it is very difficult to give a relatively good initial value of the parameters. The PSO algorithm, which works on the social behavior of particles in a swarm [22], can be used to solve the optimization problem without the initial parameter guesses. We only need to provide the range of solution space (or the range of the parameters). All the particles are considered a swarm, and each particle flies around in the solution space. The particle's position is dynamically updated according to its own experience and the swarm's experience in the iterative process $[23,24]$. The advantages of the PSO algorithm are that it converges rapidly and it has few adjustable parameters. It should be noted that the optimization algorithm may converge to the local minimum and cannot give the optimal solution. Thus, we define the quality index, which is used as an indicator for determining whether the calculated solution is a global optimal solution. In order to better understand the PSO algorithm, the detailed descriptions of some key terms in the PSO algorithm are given as follows.

(1) Particle flies in the solution space. It has two attributes, position and velocity.

(2) Position denotes the candidate solution for the optimization problem. It is dynamically updated for finding the optimal solution according to the velocity.

(3) Velocity determines the flight direction and distance of the particle in the next step. It is dynamically updated according to the particle's best position and the swarm's best position.

(4) Fitness function is a function for describing optimization problem. It is used to evaluate the position of each particle.

(5) Fitness is the value of the fitness function with the particle's position. It is used to reflect the quality of the position in the optimization.

More details of the PSO algorithm can be seen in $[22,25,26]$. Here, we develop a dedicated PSO algorithm for tracking the target based on a scalar magnetometer array and give the pseudocode of the algorithm.

In the algorithm, the parameter $w$ called inertia weight is used to bring about a balance between the global and local search. When the value of $w$ is larger, the algorithm is easier to find the global optimal solution, but converges slowly. On the contrary, when the value of $w$ is smaller, the algorithm converges faster, but is easier to fall into local optimal solution area. Thus, the value of $w$ is dynamically adjusted during the iteration. The parameters $c_{1}$ and $c_{2}$, called acceleration factors, are used to control the flight of the particle to the particle's best position and the swarm's best position. Researchers have done much work on how to select the ideal parameters for PSO implementation [27-29]. In [28, 29], inertia weight is suggested to be dynamically adjusted from 


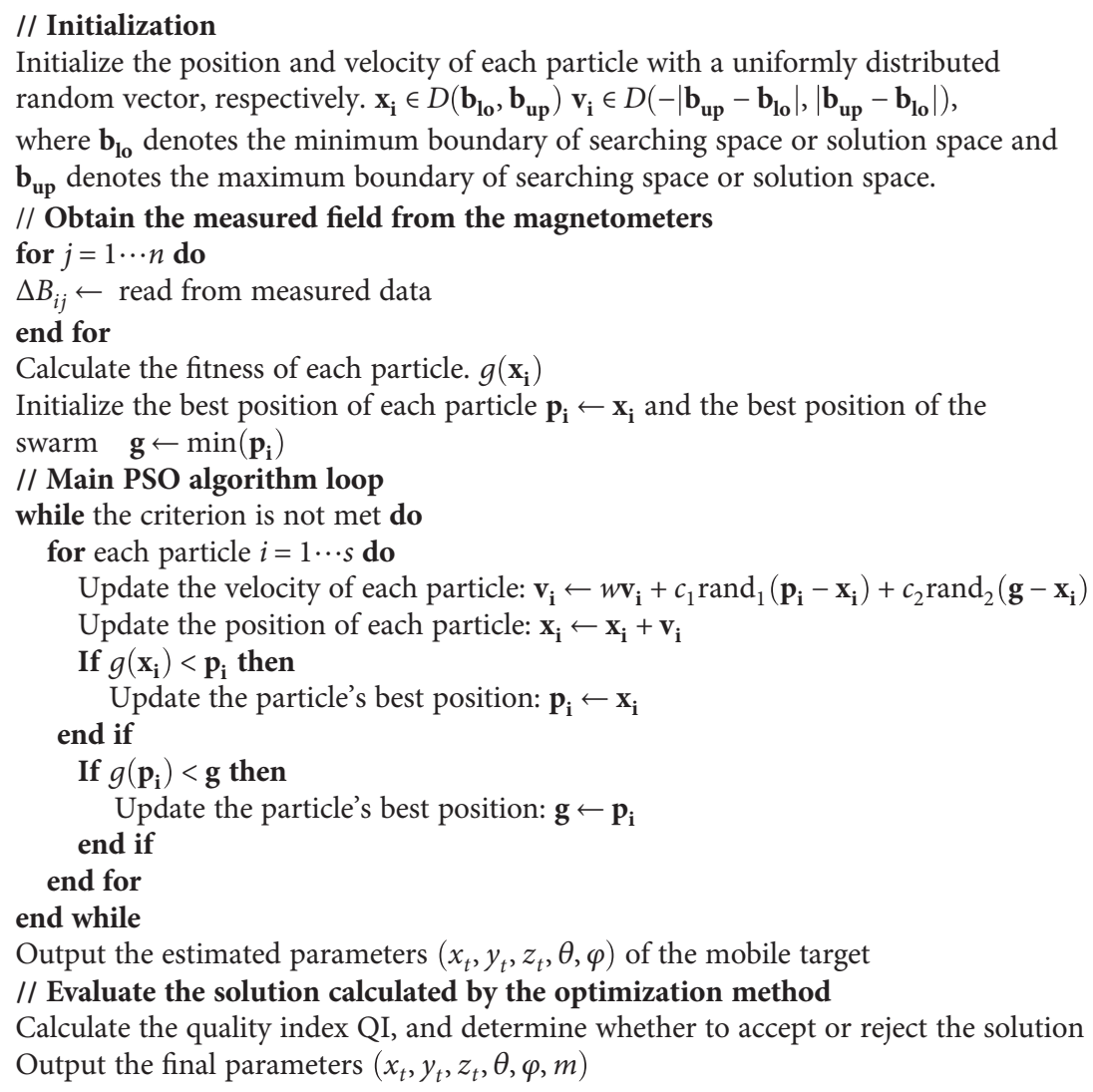

Algorithm 1: PSO algorithm pseudocode.

0.9 at the beginning to 0.4 at the end of search, and acceleration factors are suggested to be constant as 1.49 or 2. In addition, the particle's population size $s$ can affect the accuracy and efficiency of the algorithm. It should be determined according to the complexity of the fitness function. The execution time of the PSO algorithm increases along with the increase of the population size. In general, the execution time of the PSO algorithm may be longer than that of the L-M algorithm.

\section{Simulation Section}

In simulation, the proposed method was applied to track the magnetic target. The parameters of a magnetic target were set as $(m, \theta, \varphi)=\left(600 \mathrm{Am}^{2}, 60 \mathrm{deg}, 15 \mathrm{deg}\right)$. The target moved on a path which was parallel to the X-axis, starting at $(-10 \mathrm{~m}, 10 \mathrm{~m},-1 \mathrm{~m})$ and ending at $(10 \mathrm{~m}, 10 \mathrm{~m},-1 \mathrm{~m})$, shown in Figure 2. The array consisted of seven sensors. One sensor was located at the origin of the coordinate system, and other sensors were located on the orthogonal axes at $2 \mathrm{~m}$ from the origin. The measurement error was set as follows: the average is zero, and the standard deviation is $0.04 \mathrm{nT}$. In the PSO algorithm, we set $c_{1}=2, c_{2}=2$, the population size to be 150, and the max iterations to be 200 .

Then, we used the proposed method to track the magnetic target. The tracking results are shown in Figures 3-7. The positions of the target were calculated by the proposed

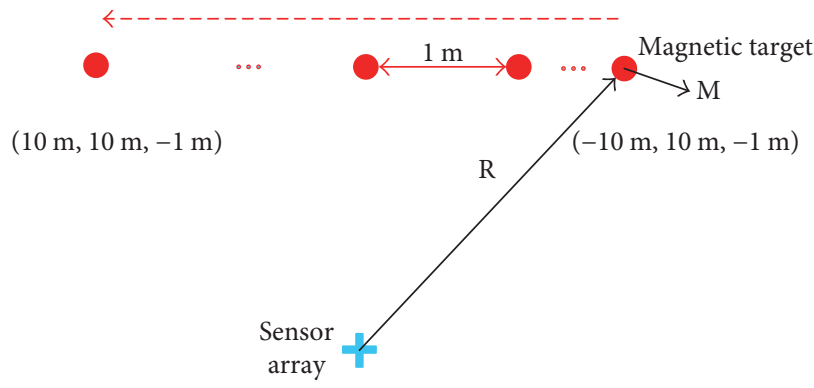

FIGURE 2: Schematic diagram of tracking simulation.

method and the normal method, shown in Figure 3. It can be seen that the calculated positions were close to the true values. The magnetic anomaly measured by the magnetometers decreased with the cube of the distance, leading to a reduction in the signal-to-noise ratio (SNR). Thus, the location accuracy decreases as the distance increases. Compared to the normal method, the proposed method had better location accuracy when the distance was large. The angles of the magnetic moment were calculated, shown in Figures 4 and 5. It can be seen that the results calculated by the proposed method are better than those by the normal method. The magnitude of the target's magnetic moment is shown in Figure 6 . The results show that the calculated values by the proposed method were closer to the true values. 


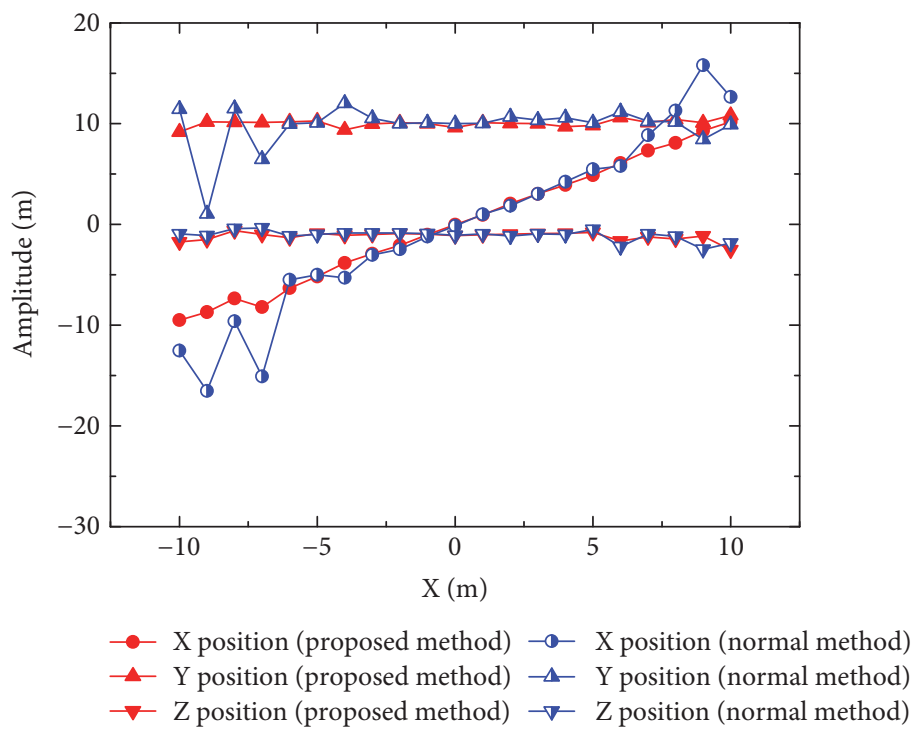

Figure 3: Estimated position of the target.

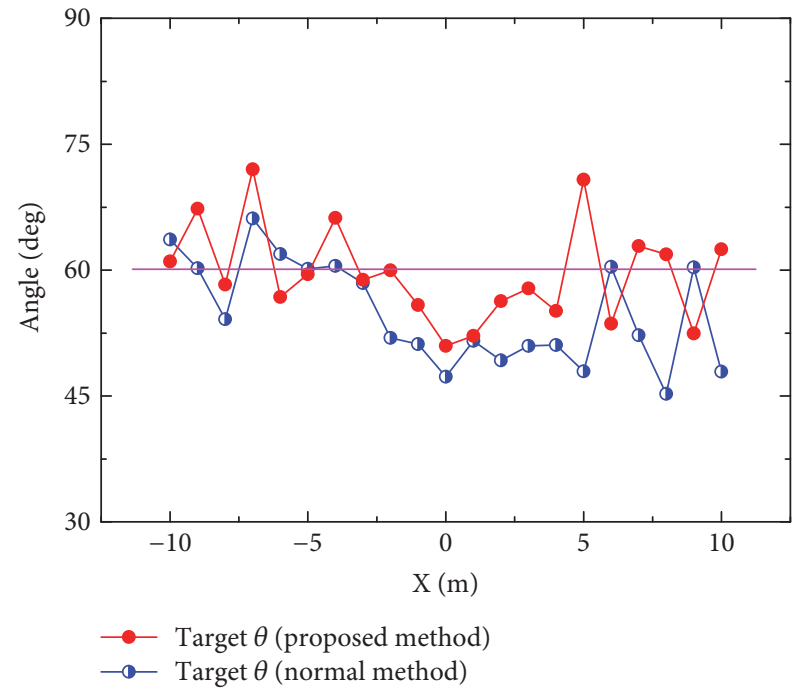

Figure 4: Estimated angle $\theta$ of magnetic moment. The solid line represents the true value.

The quality index was calculated according to (10), shown in Figure 7. It can be seen from Figure 7 that the value of the quality index was small when the solution error of the target was large. Therefore, the quality index can be used to estimate the accuracy of the solution calculated by the dedicated PSO algorithm.

The quality index is used to determine whether the solution is the global optimal solution or the local optimal solution. As we know, the relative error of the global optimal solution is smaller than that of the local optimal solution. In practice, the distance $\|r\|$ and the magnitude of the magnetic moment are the major parameters for the tracking system. Thus, the calculated solution can be considered the global optimal solution if the relative error of the distance was less

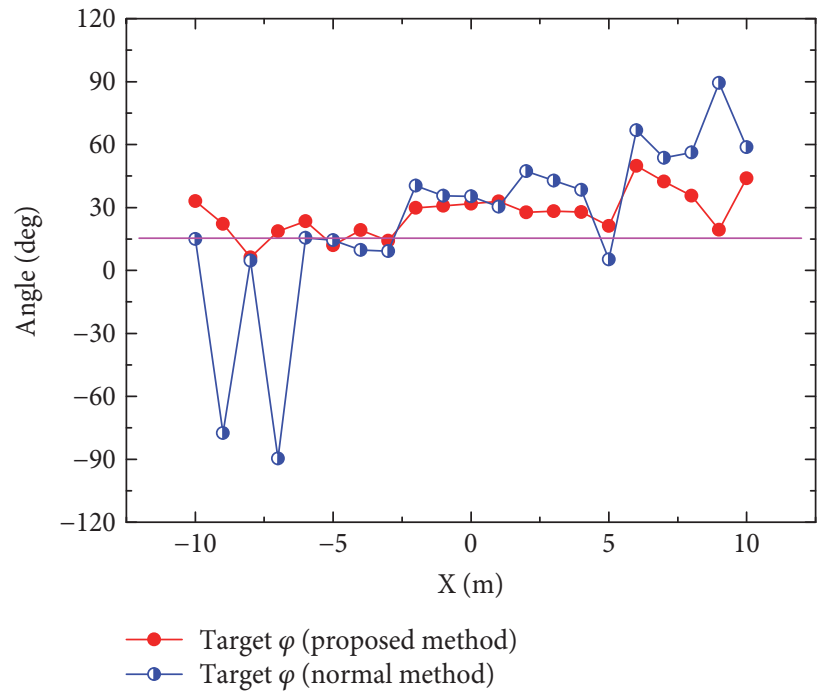

FIgURE 5: Estimated angle $\varphi$ of magnetic moment. The solid line represents the true value.

than $15 \%$ and the relative error of the moment was less than $50 \%$ in the simulation. In this case, we can determine the optimal value of the quality index, which can be used as the threshold value. After many simulations at different points, we determined that the optimal value of the quality index was 0.3 .

\section{Experimental Section}

5.1. Experimental Setup. In order to test the performance of the proposed method in practice, we used it to track a car with a constant velocity which moved along a planned trajectory. The experiment was carried out in Jinshatan Wetland Park in Harbin City, China, where the ambient magnetic 


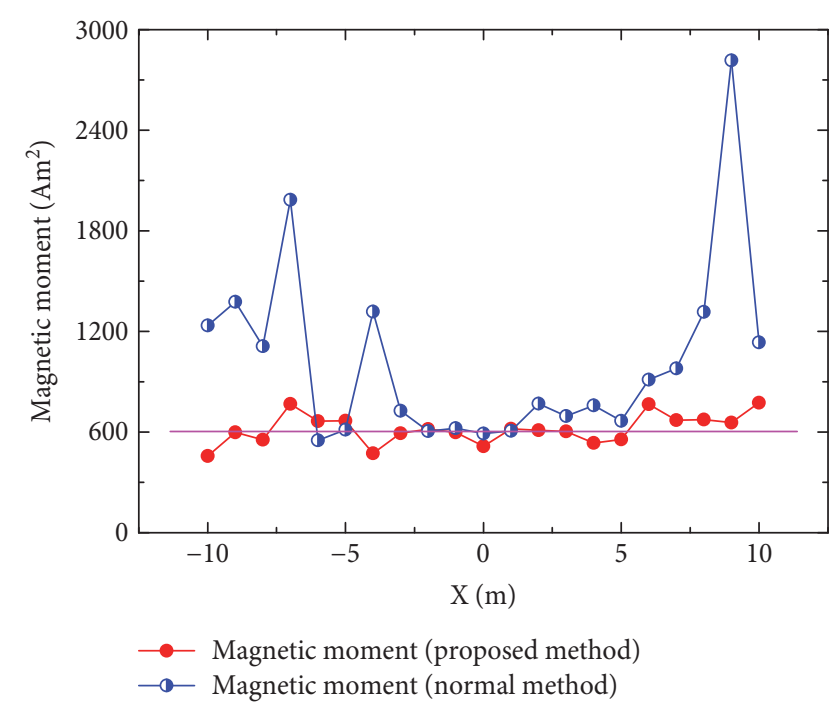

Figure 6: Estimated magnitude of the target's magnetic moment. The solid line represents the true value.

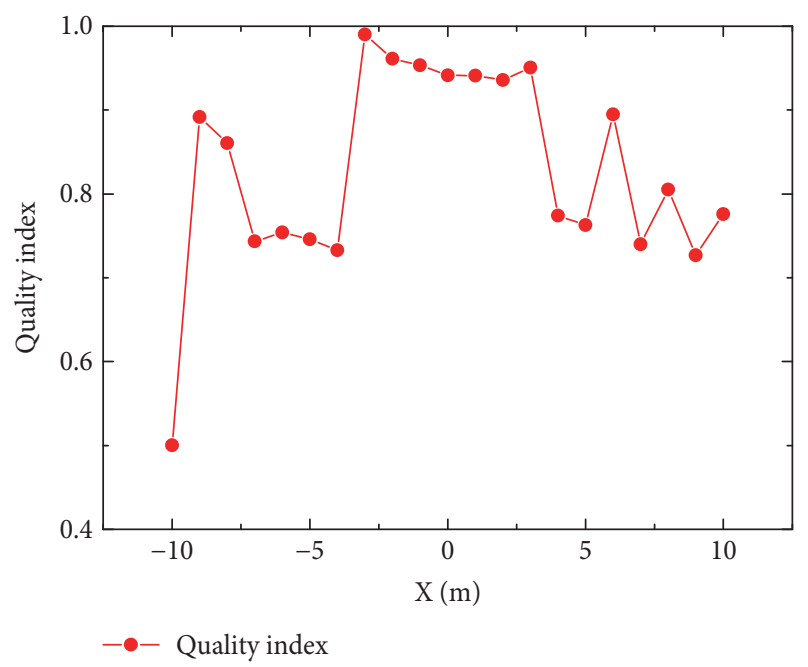

Figure 7: Quality index of the solution calculated by the proposed method.

activity due to external sources, such as power lines and traffic, was very low. We used the north-east-down (NED) coordinate system in the experiment and located the center of the array at the origin in the experiment. The array consisted of four optically pumped cesium magnetometers (CS-L [Scintrex, Snidercroft Road, Concord, Ontario, Canada]), and each magnetometer was located on the orthogonal axes at $2 \mathrm{~m}$ from the origin, as shown in Figure 8. This magnetometer has high sensitivity, and intrinsic noise is about $0.6 \mathrm{pT} / \sqrt{\mathrm{Hz}}$ at $1 \mathrm{~Hz}$. In addition, there is also another source of magnetic reading uncertainty in cesium vapour magnetometers called the heading error $( \pm 0.2 \mathrm{nT}$ for CS-L). It is a function of the angle of the sensor head with respect to the local magnetic field. In this experiment, we used a static sensor array to track the

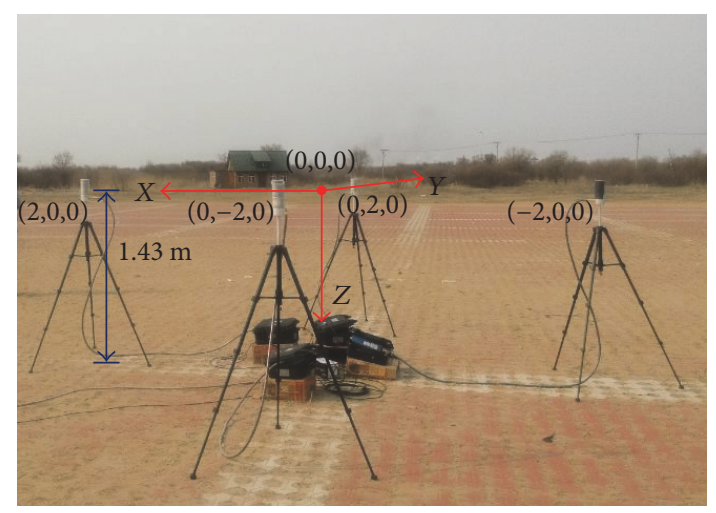

FIGURE 8: Scalar magnetometer array with four optically pumped magnetometers.

magnetic target. We did our best to ensure that the magnetometers had the same orientation so that they would have the same heading error. Thus, the heading error was not considered in this experiment. The measured data were acquired at a sampling rate of $2 \mathrm{~Hz}$.

An SUV car as the target was driven along the planned trajectory in the horizontal plane. The plan trajectory was parallel to the X-axis with $y=31.05 \mathrm{~m}$. In the experiment, the target started at $x=14.0 \mathrm{~m}$ and ended at $x=14.5 \mathrm{~m}$, the $\mathrm{Z}$ position of the target was $-0.63 \mathrm{~m}$, and the parameters of magnetic moment remained constant at $\left[485.8 \mathrm{Am}^{2}, 33.8^{\circ}, 48.7^{\circ}\right]$.

5.2. Experimental Results. Since there were four optically pumped magnetometers in the experiment, we can only obtain three unrelated magnetic anomalies $\Delta B_{i j}$. We can thus estimate only three parameters of the target. Therefore, we set the parameters $\left(z_{t}, \theta, \varphi\right)$ and the magnetic target as known values and applied the proposed method on the real data measured by the magnetometers to estimate the other three parameters $\left(x_{t}, y_{t}, m\right)$.

The results are shown in Figures 9-11. The estimated positions of the target moving in the X-Y plane are shown in Figure 9, and they are seen to be close to the true values. The localization error at both ends of the trajectory was large compared with that at the middle, because the signal-to-noise ratio (SNR) at the ends was smaller than that at the middle.

After we obtained the optimal solution about parameters $\left(x_{t}, y_{t}\right)$ of the target by the dedicated PSO algorithm, we can use (8) to calculate the magnitude of the magnetic moment. The average magnitude of the magnetic moment of the target was estimated by the magnetic anomaly $\Delta B_{i j}$, as shown in Figure 10. Due to the localization error, the error of the magnetic moment was large. Then, we used the quality index defined in (10) to evaluate the quality of the optimal solution calculated by the proposed method. We can directly determine that the solution was accepted or rejected by the quality index. From (10), the solution is good if the value of the quality index is near 1 and the solution is bad if the value of the quality index is near 0 . The threshold value of the 


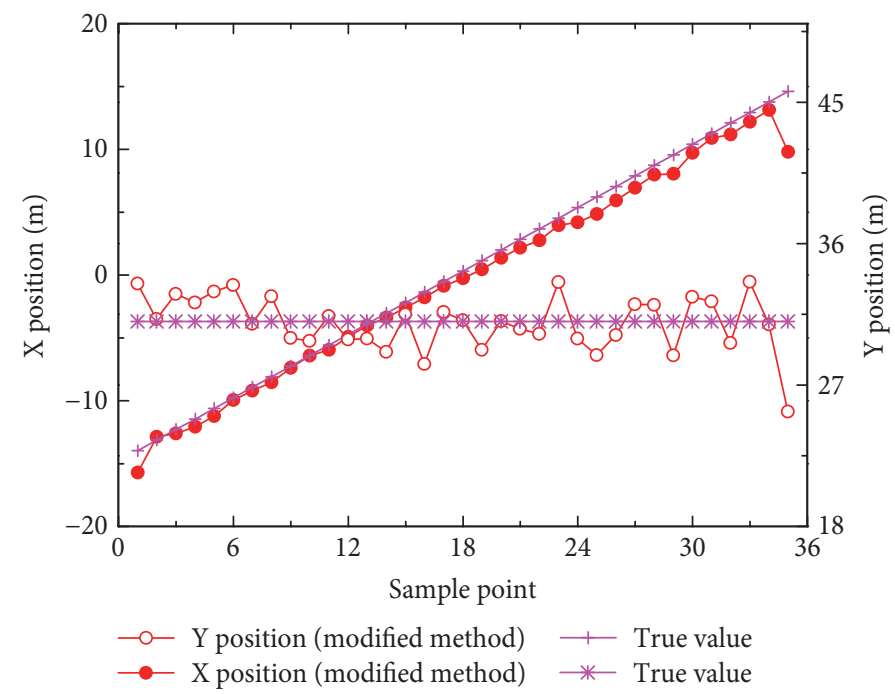

Figure 9: Position of the moving target in the X-Y plane was estimated by the proposed method.

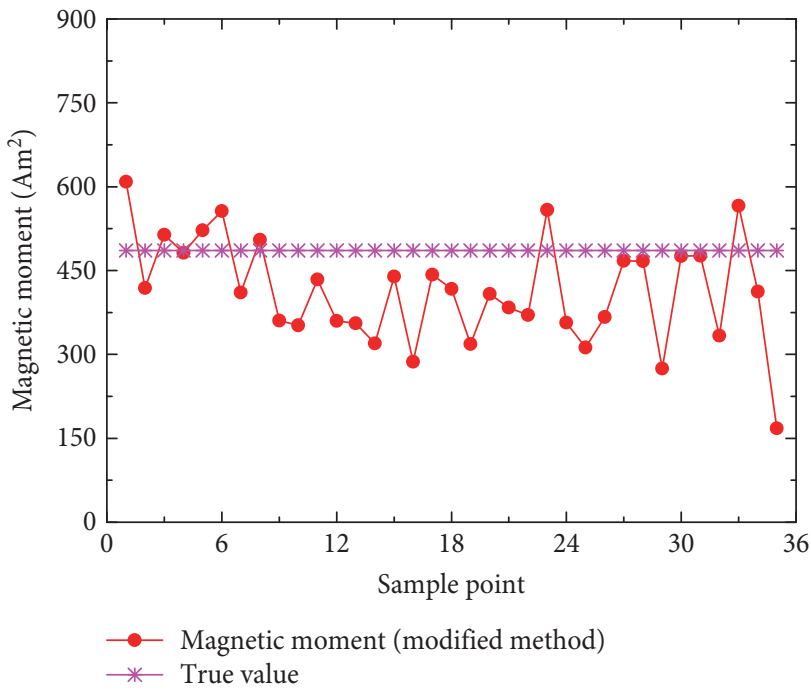

Figure 10: Magnitude of the target's magnetic moment.

quality index for accepting or rejecting the solution should be determined based on the positioning accuracy requirement. The quality index of the solution is shown in Figure 11. We can see that the value of the quality index was small at both ends. It means that the localization error was relatively larger.

\section{Conclusions}

In this paper, we presented a method based on a scalar magnetometer array to track a moving magnetic target. In this method, we isolated the magnitude of the magnetic moment from the scalar magnetic anomaly and formulated an optimization problem based on it to determine the position and magnetic parameters of the target. Then, a dedicated particle swarm optimization algorithm was implemented to solve this

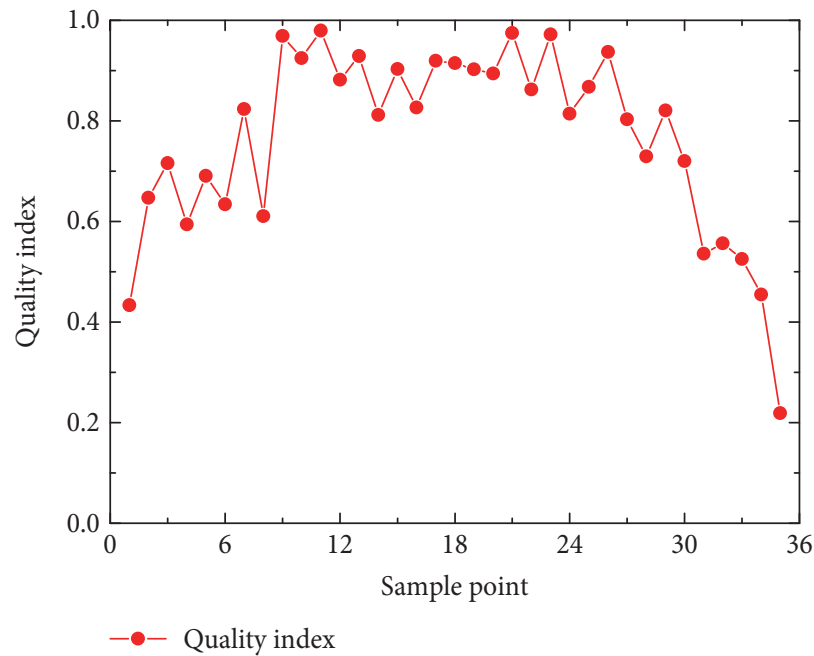

Figure 11: Quality index of the solution calculated by the proposed method.

optimization problem. The PSO algorithm may converge to the local minimum and cannot give the optimal solution. Thus, we define the quality index, which is used as an indicator for determining whether the calculated solution is a global optimal solution. We can improve the accuracy of the tracking of the remote magnetic target based on the quality index. The proposed method was applied to the simulation and the real data to track the magnetic target. The results showed that the tracked trajectory was very close to the true trajectory.

\section{Conflicts of Interest}

The authors declare that they have no conflict of interests. 


\section{Acknowledgments}

This work was supported by the National Natural Science Foundation of China (no. 61174192).

\section{References}

[1] W. Wynn, "Magnetic dipole localization using the gradient rate tensor measured by a five-axis magnetic gradiometer with known velocity," in SPIE's 1995 Symposium on OE/Aerospace Sensing and Dual Use Photonics. International Society for Optics and Photonics, pp. 357-367, 1995.

[2] T. Nara, S. Suzuki, and S. Ando, "A closed-form formula for magnetic dipole localization by measurement of its magnetic field and spatial gradients," IEEE Transactions on Magnetics, vol. 42, no. 10, pp. 3291-3293, 2006.

[3] G. Iacca, F. L. Bakker, and B. H. Wortche, "Real-time magnetic dipole detection with single particle optimization," Applied Soft Computing, vol. 23, pp. 460-473, 2014.

[4] M. Birsan, "Recursive Bayesian method for magnetic dipole tracking with a tensor gradiometer," IEEE Transactions on Magnetics, vol. 47, no. 2, pp. 409-415, 2011.

[5] Y. Gang, Z. Yingtang, L. Zhining, F. Hongbo, and R. Guoquan, "Detection of ferromagnetic target based on mobile magnetic gradient tensor system," Journal of Magnetism and Magnetic Materials, vol. 402, pp. 1-7, 2016.

[6] N. Wahlstrom and F. Gustafsson, "Magnetometer modeling and validation for tracking metallic targets," IEEE Transactions on Signal Processing, vol. 62, no. 3, pp. 545-556, 2014.

[7] J. McFee and Y. Das, "Determination of the parameters of a dipole by measurement of its magnetic field," IEEE Transactions on Antennas and Propagation, vol. 29, no. 2, pp. 282287, 1981.

[8] Z. Zalevsky, Y. Bregman, N. Salomonski, and H. Zafrir, "Resolution enhanced magnetic sensing system for wide coverage real time UXO detection," Journal of Applied Geophysics, vol. 84, pp. 70-76, 2012.

[9] E. Abdelrahman and K. Essa, "A new method for depth and shape determinations from magnetic data," Pure and Applied Geophysics, vol. 172, no. 2, pp. 439-460, 2015.

[10] R. Liu and H. Wang, "Detection and localization of improvised explosive devices based on 3-axis magnetic sensor array system," Procedia Engineering, vol. 7, pp. 1-9, 2010.

[11] Y. Sui, G. Li, S. Wang, and J. Lin, "Asphericity errors correction of magnetic gradient tensor invariants method for magnetic dipole localization," IEEE Transactions on Magnetics, vol. 48, no. 12, pp. 4701-4706, 2012.

[12] J. E. McGary, "Real-time tumor tracking for four-dimensional computed tomography using SQUID magnetometers," IEEE Transactions on Magnetics, vol. 45, no. 9, pp. 3351-3361, 2009.

[13] J. Lenz and S. Edelstein, "Magnetic sensors and their applications," IEEE Sensors Journal, vol. 6, no. 3, pp. 631-649, 2006.

[14] A. B. Reid, J. Allsop, H. Granser, A. T. Millett, and I. Somerton, "Magnetic interpretation in three dimensions using Euler deconvolution," Geophysics, vol. 55, no. 1, pp. 80-91, 1990.

[15] M. Mushayandebvu, V. Lesur, A. Reid, and J. Fairhead, "Grid Euler deconvolution with constraints for 2D structures," Geophysics, vol. 69, no. 2, pp. 489-496, 2004.

[16] P. Keating and M. Pilkington, "Euler deconvolution of the analytic signal and its application to magnetic interpretation," Geophysical Prospecting, vol. 52, no. 3, pp. 165-182, 2004.
[17] D. Thompson, "EULDPH: a new technique for making computer-assisted depth estimates from magnetic data," Geophysics, vol. 47, no. 1, pp. 31-37, 1982.

[18] W. M. Wynn, "Detection, localization, and characterization of static magnetic dipole sources, detection and identification of visually obscured," Targets, pp. 337-374, 1999.

[19] A. Sheinker, B. Lerner, N. Salomonski, B. Ginzburg, L. Frumkis, and B.-Z. Kaplan, "Localization and magnetic moment estimation of a ferromagnetic target by simulated annealing," Measurement Science and Technology, vol. 18, no. 11, pp. 3451-3457, 2007.

[20] R. Alimi, E. Weiss, T. Ram-Cohen, N. Geron, and I. Yogev, “A dedicated genetic algorithm for localization of moving magnetic objects," Sensors, vol. 15, no. 9, pp. 23788-23804, 2015.

[21] C. Hu, M. Q.-H. Meng, S. Song, and H. Dai, "A sixdimensional magnetic localization algorithm for a rectangular magnet objective based on a particle swarm optimizer," IEEE Transactions on Magnetics, vol. 45, pp. 3092-3099, 2009.

[22] R. C. Eberhart and J. Kennedy, "A new optimizer using particle swarm theory," in MHS'95. Proceedings of the Sixth International Symposium on Micro Machine and Human Science, pp. 39-43, Nagoya, Japan, 1995.

[23] A. Ratnaweera, S. K. Halgamuge, and H. C. Watson, "Self-organizing hierarchical particle swarm optimizer with time-varying acceleration coefficients," IEEE Transactions on Evolutionary Computation, vol. 8, no. 3, pp. 240-255, 2004.

[24] J. Robinson and Y. Rahmat-Samii, "Particle swarm optimization in electromagnetics," IEEE Transactions on Antennas and Propagation, vol. 52, no. 2, pp. 397-407, 2004.

[25] S.-W. Lin, K.-C. Ying, S.-C. Chen, and Z.-J. Lee, "Particle swarm optimization for parameter determination and feature selection of support vector machines," Expert Systems with Applications, vol. 35, no. 4, pp. 1817-1824, 2008.

[26] W. A. Yang, C. Hu, M. Li, M. Q. H. Meng, and S. A. Song, "A new tracking system for three magnetic objectives," IEEE Transactions on Magnetics, vol. 46, no. 12, pp. 4023-4029, 2010.

[27] Y. Shi and R. C. Eberhart, "Parameter selection in particle swarm optimization," in International Conference on Evolutionary Programming, pp. 591-600, Springer, 1998.

[28] Y. Shi and R. C. Eberhart, "Empirical study of particle swarm optimization," in Proceedings of the 1999 Congress on Evolutionary Computation-CEC99 (Cat. No. 99TH8406), Washington, DC, USA, 1999.

[29] Y. Shi, "Particle swarm optimization: developments, applications and resources," in Proceedings of the 2001 Congress on Evolutionary Computation (IEEE Cat. No. 01TH8546), pp. 81-86, Seoul, South Korea, 2001. 


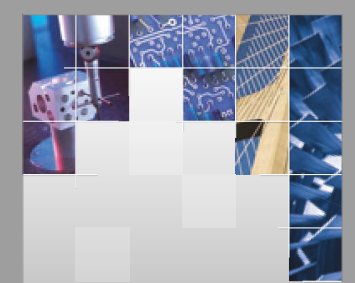

\section{Enfincering}
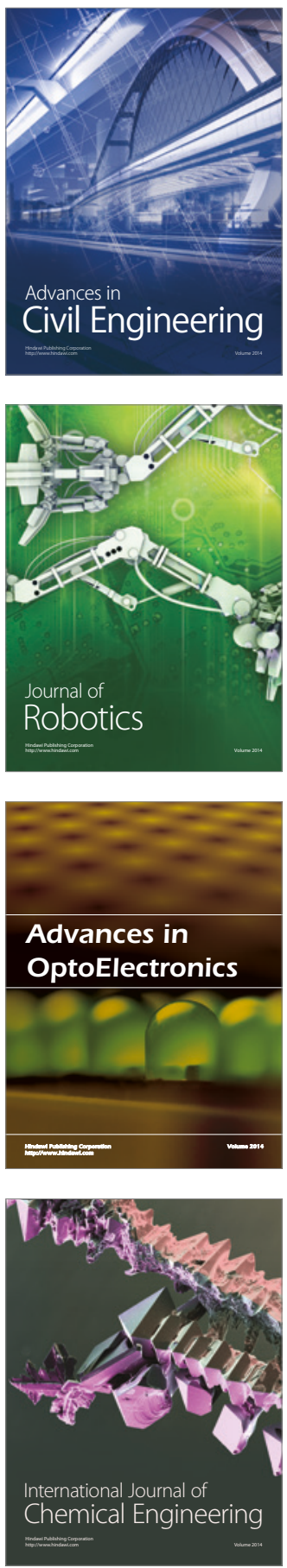

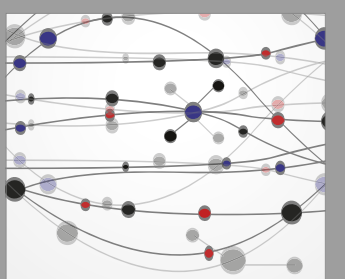

The Scientific World Journal

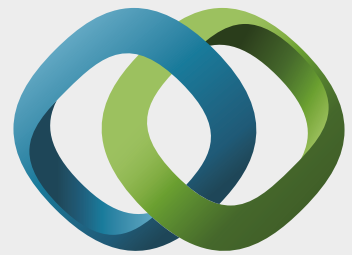

\section{Hindawi}

Submit your manuscripts at

https://www.hindawi.com
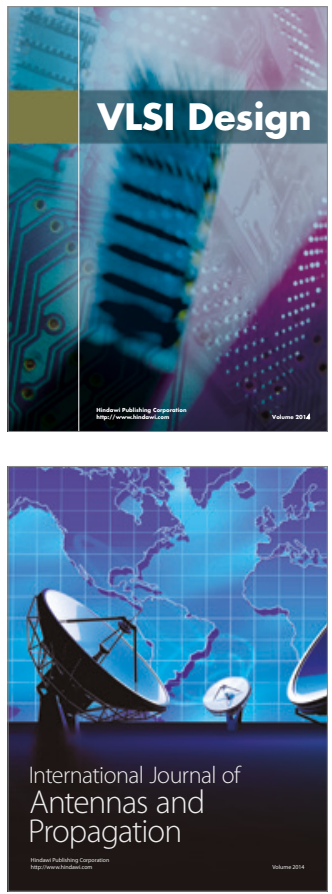

\section{Rotating}

Machinery
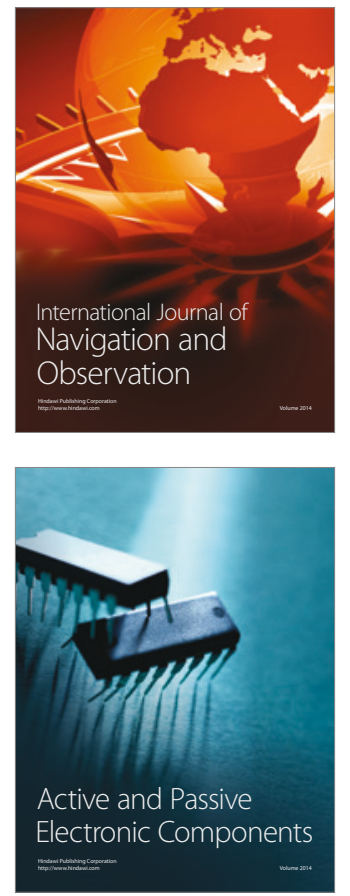
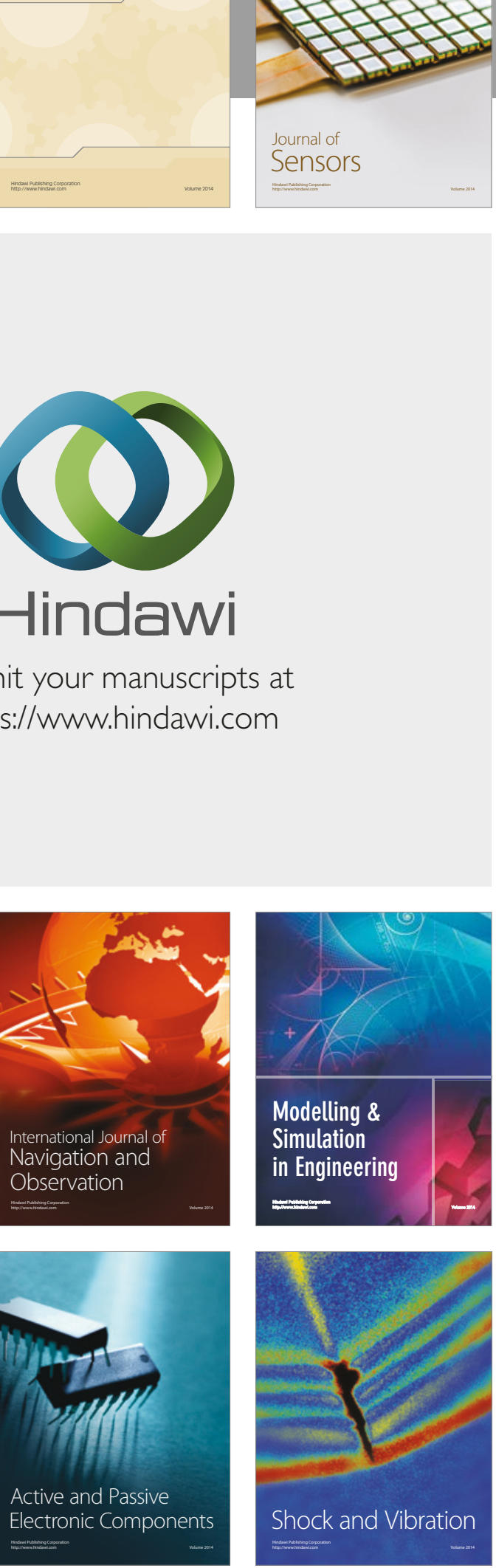
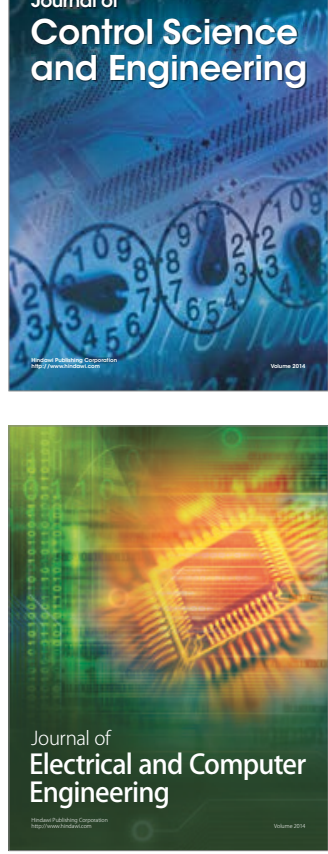

Distributed

Journal of

Control Science

and Engineering
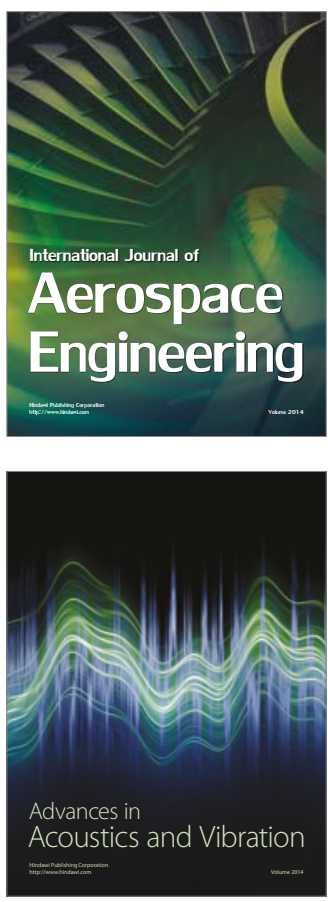

Sensor Networks 